\title{
Presence of a Non-HLA B Cell Antigen in Rheumatic Fever Patients and Their Families as Defined by a Monoclonal Antibody
}

\author{
Ashwani K. Khanna," Daniel R. Buskirk, " Ralph C. Williams, Jr., Allan Gibofsky," Mary K. Crow," Arvind Menon," \\ Marilena Fotino," Hugo M. Reid," Theo Poon-King," Pablo Rubinstein,"* and John B. Zabriskie* \\ *The Rockefeller University, New York 10021; ${ }^{\ddagger}$ University of Florida, Gainesville, Florida $32610 ;{ }^{\S}$ Hospital for Special Surgery, \\ New York 10021; "Rogosin Institute, New York 10021; 'San Fernando Hospital, Trinidad, West Indies; \\ **New York Blood Center, New York 10021
}

\begin{abstract}
Numerous investigators have suspected that there is a genetic predisposition to rheumatic fever (RF). In this context we have recently produced a series of monoclonal antibodies directed against $B$ cells obtained from RF patients one of which, labeled D8/17, identifies a B cell antigen present in $100 \%$ of all $\mathbf{R F}$ patients studied. While the highest percentage of positive cells were exhibited by RF probands $(33.5 \% \pm \mathrm{SE})$, the percentage of cells in unaffected siblings and parents was 14.6 and $13 \%$, respectively. The percentage of positive cells in APSGN probands, unaffected siblings, and parents was $2.96,3.86$, and $2.8 \%$, respectively. A low level of B cells (5-7\%) bearing the D8/17 marker was seen in control patients.

The segregation pattern of the phenotypes defined by the percentage of D8/17 positive cells within HLA-typed RF families are consistent with an autosomal recessive mode of inheritance not associated with the human MHC system. We postulate that these phenotypes indicate the presence of at least one necessary genetic factor for susceptibility to RF.
\end{abstract}

\section{Introduction}

Although thought to be on the decline for many years, rheumatic fever (RF) ${ }^{1}$ appears to be recurring as an important public health problem in the United States. Recently, several outbreaks have been reported $(1,2)$ that were unassociated with any of the previously presumed risk factors such as poor socioeconomic conditions, hygiene, or substandard medical care. These recent observations suggest that other factors, possibly including genetic predisposition of the host to contract the disease, might play an important role in the evolution of the disease. This hypothesis is compatible with a large body of evidence (reviewed in reference 3 ) demonstrating that there are abnormal cellular and humoral responses on the part of the host to a number of streptococcal antigens cross-reactive with mammalian tissue that are not seen in normal individuals or unaffected siblings of the patient.

Address reprint requests to Dr. J. B. Zabriskie, The Rockefeller University, 1230 York Avenue, New York, NY 10021-6399.

Received for publication 1 April 1988 and in revised form 19 December 1988.

1. Abbreviation used in this paper: RF, rheumatic fever.

J. Clin. Invest.

(c) The American Society for Clinical Investigation, Inc.

0021-9738/89/05/1710/07 \$2.00

Volume 83, May 1989, 1710-1716
The concept that RF might be the result of a genetic predisposition has intrigued investigators since Cheadle (4) first noted an increased familial incidence of RF. Several subsequent family studies also suggested a genetic susceptibility to the disease, but the investigators disagreed concerning the mode of inheritance. For example, Wilson et al. (5) suggested that susceptibility to RF might be the result of the inheritance of a single gene in an autosomal recessive fashion. In contrast, Taranta et al. (6), based on his twin studies, postulated that the genetic factor might have limited penetrance since he observed a lower than predicted concordance rate in monozygotic twins.

Renewed interest in the genetic aspects of RF occurred with the recognition that gene products of the human major histocompatibility system (MHC) are significantly associated with a number of autoimmune and rheumatic diseases. To date, however, a number of studies of HLA antigens in patients with RF have yielded inconsistent and conflicting results $(7,8)$. The recent studies of Ayoub et al. (9) have helped to resolve some of the discrepancies in that the frequency of HLA type DR4 was increased in Caucasian RF patients while DR2 was significantly increased in Black patients emphasizing the importance of the study of specific populations of RF patients.

In contrast to the suggested associations with known HLA antigens, Patarroyo et al. (10) reported that a serum from a multiparous woman identified a novel $B$ cell alloantigen marker, 883 , in individuals previously identified as having had RF with or without subsequent rheumatic heart disease. As reported, $71 \%$ of patients typed in New York and $74 \%$ of patients typed in Bogota, Colombia, South America expressed this antigen on their B cells as compared with a frequency of only $17 \%$ in the two disease-free control groups tested. An important feature of the marker was the observation that this B cell alloantigen did not appear to be associated with any of the known MHC antigens. In a subsequent study, Zabriskie et al. (11) produced monoclonal antibodies by immunization of mice with $B$ cells from known $883^{+}$and $883^{-}$inactive RF patients described in the original study. Two monoclonal antibodies resulted which, when used in combination, identified $92 \%$ of patients, as compared with $\sim 21 \%$ of disease-free controls selected from the same general ethnic population pool as the RF patients.

Recently, we have developed a third monoclonal antibody, D8/17, which appears to recognize a cell-surface marker common to both $883^{+}$and $883^{-}$rheumatics, as defined in our original studies. This report examines the level of expression of the $B$ cell antigen identified by the D8/17 antibody in RF patients and their families and in patients and their relatives with acute poststreptococcal glomerulonephritis. Controls used were disease-free individuals matched for age, sex, and 
racial background to the RF patients as well as nephritic patients and family members from the same racial background.

\section{Methods}

Patient selection. Patients with RF/rheumatic heart disease were seen either at the Rheumatic Fever Clinic of The Rockefeller University Hospital, the University of New Mexico at Albuquerque, the University of Utah or the San Fernando Hospital, Trinidad, West Indies. All patients fulfilled the modified Jones criteria (12) for the original diagnosis of acute RF at the time of the original attack. In order not to introduce selection bias in our studies, patients were tested sequentially for the D8/17 marker during their routine followup visits to their respective clinics. In addition to the New York families, a selected number of RF patients as well as a number of poststreptococcal nephritis families were also obtained from Trinidad, West Indies. The latter group were matched for the same East Indian background. All RF and nephritic patients were clinically inactive and were studied $2-25 \mathrm{yr}$ after the initial diagnosis. 76 disease-free individuals served as controls and were drawn from the same ethnic population as the patients in each of the different geographical locations.

Monoclonal antibodies. Monoclonal antibodies to B cells isolated from RF were produced as previously described (11). Briefly, $100 \mathrm{~cm}^{3}$ of heparinized blood was drawn from both $883^{+}$or $883^{-} \mathrm{RF}$ individuals so defined by the original alloantigen; the $B$ cells were isolated as described and mice were injected twice one month apart with $2 \times 10^{7}$ cells per mouse each time. The fusion occurred $4 \mathrm{~d}$ after the last injection. The D8/17 antibody (a murine IgM antibody) described below was derived from a fusion obtained following the injection of $B$ cells from a patient who had had well documented RF 20 years ago and who presently has rheumatic heart disease with mitral valvular damage.

Screening of monoclonal antibodies. Monoclonal antibodies were screened by immunofluorescence first against $\mathrm{KH}$ cells (a human lymphoblastoid cell line known from previous studies (10) to express the $883^{+}$antigen) then against $B$ cells and other cell types (e.g., $T$ cells, macrophages, non-B cells) obtained from both $883^{+}$and $883^{-}$RF patients and disease-free controls who were identified in previous studies (10). Additional controls were obtained from disease free individuals of similar racial backgrounds in Utah and New Mexico.

Separation of cell subpopulations. Mononuclear cell preparations were prepared from heparinized blood using the Ficoll-hypaque separation method of Boyum (13). The cells were counted and mixed with a $10 \%$ suspension of neuraminidase treated sheep red blood cells prepared by the method of van de Putte et al. (14) at a ratio of 30 million mononuclear cells to $1 \mathrm{ml}$ of sheep red blood cells. The cell mixture was first incubated at $37^{\circ} \mathrm{C}$ for $8-10 \mathrm{~min}$, centrifuged at $200 \mathrm{~g}$ for $8-10$ min and then placed at $4^{\circ} \mathrm{C}$ overnight. The following day, the B cells were isolated using Ficoll-hypaque differential centifugation. The nonrosette forming B cell enriched population was collected from the interface. The precipitate, consisting of sheep red blood cells rosetted T cells, was washed and the red blood cells lysed with $0.17 \mathrm{M} \mathrm{NH}_{4} \mathrm{Cl}$ solution. The resulting $\mathrm{T}$ cells were washed and adjusted to the desired concentration for the immunofluorescence tests. In selected experiments the B cell-enriched populations were further separated by density utilizing a Percoll gradient technique previously described (15).

$B$ cell activation antigen studies. The B cell-enriched populations as described above were obtained from rheumatics exhibiting elevated levels of positive B cells with MAb D8/17, or normals with low values of $D 8 / 17$ positive cells. These cells $(100,000 /$ sample) were incubated for $1 \mathrm{~h}$ in either PBS with $1 \%$ BSA or the appropriate monoclonal antibody specific for the $B$ cell activation antigen. The cells were then washed three times in PBS - $1 \%$ BSA solution and labeled with either fluorescence-conjugated $\mathrm{F}\left(\mathrm{Ab}^{\prime}\right)_{2}$ goat anti IgG or IgM depending on the isotype of the monoclonal antibody used. Immunofluorescence was assayed by FACS analysis. Background fluorescence was subtracted from the results for each antibody.
The source of the monoclonal antibodies was as follows: Anti-Tac MAb was obtained from Dr. Thomas Waldmann (National Institutes of Health), Anti-4F2 MAb from Dr. Alice Gottleib (The Rockefeller University), and Anti-CD23 MAb from Drs. Stan Metzenberg and Bill Sudgen (McCardle Institute, Madison, WI).

Immunofluorescence for D8/17 antigen. Briefly, $1.5 \times 10^{5}$ cells $(B$ or T or KH cells) in $100 \mu \mathrm{l}$ were placed in wells of a 96-well plate (Falcon 3911; microtest plate Falcon Labware), centrifuged, and washed three times with PBS-BSA buffer (PBS with $1 \%$ BSA and $0.1 \%$ sodium azide). $50 \mu \mathrm{l}$ of the monoclonal antibody designated D8/17 was added to each well, mixed thoroughly, and the plate incubated at $4^{\circ} \mathrm{C}$ for $1 \mathrm{~h}$. The cells were again washed three times with PBS-BSA buffer, incubated for $1 \mathrm{~h}$ at $4^{\circ} \mathrm{C}$ with $100 \mu \mathrm{l}$ of a 1:40 dilution of FITC anti-mouse IgM (U chain specific) antibody (Tago Inc., Burlingame, CA or Cooper Biomedical, Malvern, PA) and again washed three times in PBS-BSA buffer. The cells were suspended in $15 \mu \mathrm{l}$ of buffer and mounted on a microscopic slide. The fluoresceinated cells were counted and the percentage of positive cells in the B cell-enriched mononuclear population was calculated on 500 cells for each individual. The percentage of positive $B$ cells for each individual was counted separately by two independent investigators who were unaware of the identity of the proband or members within a given family. As a further confirmation of the specificity of the reaction, both immunofluorescence and flow cytometric analysis was carried out on identical aliquots of six samples tested in Utah by FACS and in New York by fluorescence and the results were essentially identical $(36.8 \%$ and $32 \%$, respectively).

"Positive" and "negative" individuals. Each center identified the percentage of positive B cells in a slightly different manner but the results were essentially the same. For example the New York population was studied using a B cell-enriched population in which the percentage of D8/17 positive cells was counted using the FITC indirect immunofluorescence method. Dr. Williams counted the number of D8/17 positive cells as a percentage of positive $B$ cells using as a second label anti-human IgG. Dr. DeWitt examined the cells by FACS and counted the percentage of positive $\mathrm{D} 8 / 17$ cells as a percentage of the positive B cells using as a second label anti-human DR antibody.

Examination of the data of rheumatics and normals in all three centers revealed a skewness in the normal population with a majority of the negative individuals having 5-7\% D8/17 positive cells. However, there were a number of normal unaffected individuals who were above this value but did not exhibit disease.

Thus, individuals exhibiting $1+$ SD above the normal values were considered positive (i.e., $>11.8 \%$ ). Using this criterion, all but one rheumatic fever patient was positive, the exception being one individual with $11 \%$ positive cells which is within the experimental error of the test. Furthermore this designation of positive identified normal individuals as well as siblings of rheumatic fever patients who were clearly different from the normal population yet were unaffected individuals.

HLA typing. HLA typing for serologically detectable antigens of the A, B, C, DR, and DQ series was performed according to standard methods using antisera standardized as part of the 9th and 10th International Workshops (16).

Statistical methods. Chi-square and $P$ values and one-way analysis of variance were calculated according to standard methods (17).

\section{Results}

Presence of D8/17 and proportion of positive individuals in rheumatics and healthy controls. Examination of the frequency distribution of cells binding D8/17 in controls and rheumatic patients by analysis of variance (ANOVA) demonstrated a significant difference: $F(1,64)=227.3$ with $P$ $<0.0001$. Moreover, the distribution of the values in controls was markedly skewed, showing several values in the range of the patients. For this reason, the ranges of values were categorized as negative or positive depending on whether they were 
below or exceeded the mean +1 SD of the healthy controls (i.e., 11.8\%). This criterion puts all but one of the patients in the positive range, the exception having a count of $11 \%$, within the technical error margin of the cutoff value. In contrast, 10/72 controls (13.9\%) were positive (see Table I).

Distribution of D8/17 marker in rheumatic and nephritic families. In order to determine the distribution of the marker in families who had had known exposure to streptococcal infections, we tested for the presence of the marker in the index case and unaffected siblings in a selected number of RF and nephritic families. As noted in Table II, not only were all the index cases positive for the marker, a significant number of the parents and unaffected siblings were also positive for the marker. In contrast, both the index case and the siblings and parents of a number of nephritic families all had low levels of positive B cells expressing the marker suggesting that a streptococcal antigenic stimulus per se was not solely responsible for the appearance of the D8/17 marker on B cells.

Segregation of D8/17 positivity in the families of 20 rheumatic patients. This analysis was undertaken in order to investigate the possibly inherited nature of the phenotype defined above as D8/17 positive and the relation between that phenotype and the clinical diagnosis of RF. The results, summarized in Table III, indicate that a substantial proportion of the healthy siblings of patients are $\mathrm{D} 8 / 17$ positive and that the probability of being positive depends on the phenotypes of the parents.

Three types of families were encountered according to the D8/17 phenotypes of the parents: $(+x+),(+x-)$, and $(-x$ $-)$. The healthy siblings of patients belonging to the $(+X+)$ type were almost always positive ( 22 of 25 cases). The two exceptions were in the high end of the normal range, which suggests that they might represent quantitative variants of the positive type. The healthy children in families with $(+x-)$ parents segregate into essentially equal numbers with positive $(n=8)$ and negative type $(n=11)$

These two mating types, therefore, suggest that the D8/17 phenotype is an inherited trait and, if so, that its segregation may be consistent with a recessive mode of inheritance. The three negative children of the $(+x+)$ families, however, indicate that either the penetrance of the homozygotes is incomplete or that the mode of inheritance is dominant, in which case, since most of these parents should be heterozygous, one would expect up to $25 \%$ negative offspring.

The third class of families, with $(-x-)$ parents also had four of six positive healthy sibs, which suggests that the posi-

Table I. Frequency of D8/17 Positives Among Rheumatic Patients and Healthy Controls from Three U.S. Locations

\begin{tabular}{lcccccc}
\hline & \multicolumn{2}{c}{ Patients } & & \multicolumn{3}{c}{ Controls } \\
\cline { 2 - 3 } \cline { 6 - 7 } \multicolumn{1}{c}{ Region } & $n$ & \% Positive* & & $n$ & \% Positive & $P$ (difference) \\
\hline New York & 38 & 100 & & 31 & 17 & $<0.0001$ \\
New Mexico & 31 & 97 & & 30 & 10 & $<0.0001$ \\
Salt Lake City & 15 & 100 & & 15 & 17 & $<0.0001$ \\
All & 84 & 98.8 & & 76 & 14 & $<0.0001$ \\
\hline
\end{tabular}

${ }^{*} P<0.0001$ for each patient group compared to normal controls from same area.
Table II. Average Number of B Cells Expressing the RF Marker in Rheumatic Families, Nephritic Families, and Normal Controls

\begin{tabular}{lccc}
\hline \multicolumn{1}{c}{ Group } & No. studied & $\begin{array}{c}\text { B cells positive } \\
\text { mean } \pm \text { SD \% }\end{array}$ & Range \\
\hline Rheumatic families & & & \\
Index & 14 & $33.5 \pm 8.5$ & $20.0-53.6$ \\
Siblings & 33 & $14.6 \pm 6.4$ & $4.0-26.0$ \\
Mother & 14 & $14.6 \pm 5.5$ & $6.9-25.7$ \\
Father & 14 & $11.98 \pm 5.4$ & $4.5-19.0$ \\
Normal controls & & & \\
New York & 31 & & \\
New Mexico & 30 & $4.5 \pm 4.3$ & $1.0-19.3$ \\
Nephritic families & & & $<1.0-18.0$ \\
Index & 12 & $2.96 \pm 3.0$ & \\
Siblings & 22 & $3.86 \pm 4.7$ & $0.6-10.2$ \\
Mother & 9 & $3.17 \pm 4.3$ & $0.9-20.7$ \\
Father & 9 & $2.51 \pm 3.5$ & $1.2-10.9$ \\
& & & $0.6-8.7$ \\
\hline
\end{tabular}

tive phenotype was inherited by them and by the respective probands rather than "sporadic" occurrences. This type of sibship is also consistent with a recessive mode of inheritance, although the proportion of positives $(66 \%)$ is somewhat higher than would be expected under this assumption (25\%). Further support for this hypothesis is provided by the relatively high proportion (50\%) of families with the $(+x+)$ mating type (10 of 20). Given that dominant traits require a single positive parent for their occurrence in children, a lower proportion should be anticipated from the low frequency of homozygotes in the general population.

These arguments are not definitive, however, and segregation analysis of the trait taken as a quantitative variable would not be likely to be more informative because of both the small number of families studied and the phenotypic variability of expression as demonstrated by the identical twins (see Fig. 2).

Identification of the D8/17 marker as a B-cell antigen. To further identify the predominant cell population which was positive for the D8/17 marker, the non-T cell (B cell enriched) population from four patients were separately examined following differential centrifugation on a Percoll-density gradient. Of the D8/17 cells that were positive for the marker, $92 \%$ of these cells were B cells as defined by the appropriate marker compared to $T$ cells $(2.6 \pm 1.7 \%)$ or isolated macrophages $(<1 \%)$ indicating that the antigen was expressed solely on the B cells of the patients. Further confirmation was pro-

Table III. Segregation of D8/17 Phenotypes in Unaffected Siblings of Rheumatic Fever Patients

\begin{tabular}{ccrr}
\hline & & \multicolumn{2}{c}{$\begin{array}{c}\text { Unaffected siblings } \\
\text { phenotypes }\end{array}$} \\
\cline { 3 - 4 } Parent's phenotypes & No. of families & + & - \\
\hline$+\times+$ & 10 & 22 & 3 \\
$+\times(-)$ & 7 & 8 & 11 \\
$(-) \times(-)$ & 3 & 4 & 2 \\
\hline
\end{tabular}


Table IV. Cell Surface Expression of B Cell Activation Antigens in Rheumatics and Normals

\begin{tabular}{|c|c|c|c|}
\hline \multirow[t]{2}{*}{ Subjects } & \multicolumn{3}{|c|}{$\%$ of cells positive for the respective surface antigen } \\
\hline & Tac & $4 F 2$ & $C D 23$ \\
\hline \multicolumn{4}{|c|}{ Rheumatics } \\
\hline P.Y. & 8 & 15 & 3 \\
\hline M.A. & 4 & 15 & 3 \\
\hline G.K. & 10 & 16 & 2 \\
\hline \multirow[t]{2}{*}{ B.A. } & $\underline{4}$ & $\underline{16}$ & $\underline{2}$ \\
\hline & \% Av. 6.5 & 15.5 & 3.3 \\
\hline \multicolumn{4}{|l|}{ Normals } \\
\hline D.G. & 7 & 20 & 3 \\
\hline A.K. & 5 & 20 & 2 \\
\hline \multirow[t]{2}{*}{ W.R. } & $\underline{3}$ & $\underline{16}$ & $\underline{1}$ \\
\hline & \% Av. 5.0 & 18.7 & 2.0 \\
\hline
\end{tabular}

vided by two-color FACS analysis using a phycoerythrin labeled anti-human $B$ cell marker and the D8/17 indirect staining employing FITC labeled anti-murine IgM. All D8/17 positive cells were also stained by the B cell marker.

$B$ cell activation antigen studies. In order to determine whether or not the D8/17 antigen was in reality a B cell activation marker; the $B$ cell enriched population of four well documented inactive rheumatics known to be positive for the D8/17 marker (Av. $25.2 \%$ positive B cells) and three normals (Av. 5.3\% positive B cells) were tested with monoclonal antibodies specific for the following activation antigens: Tac, 4F2 and CD23. As seen in Table IV, there was no significant difference between the two groups with respect to the percent of positive cells in spite of significantly elevated levels of D8/17 positive B cells in the rheumatics.

HLA studies. Earlier data did not suggest the existence of HLA-D8/17 associations with either the categorical D8/17 phenotypes $(+$ or - ) or with the actual percentage of positive cells. As seen in Table $V$, in a small number of RF patients, there was no association of any particular HLA phenotype with the presence of the D8/17 marker. Similarly, the segregation of these traits in four informative families appeared to be unequivocally independent (Figs. 1-3).

\section{Discussion}

In previous studies a single alloantiserum called 883 identified a B cell antigen in $71-75 \%$ of RF individuals whether from New York or Bogota, Colombia (10). Following this initial observation, two monoclonal antibodies (11) were produced one of which, called 83S19.23, had specificity identical to the previously described $883^{+}$antiserum while the second antibody, labelled 56S10 identified the majority of RF individuals originally described as $\mathbf{8 8 3}$ negative patients. In contrast, the present monoclonal antibody labeled D8/17 appears to identify a cell-surface marker which shows markedly increased expression on the B cells of all individuals previously diagnosed as having had RF or rheumatic heart disease. Only $13.9 \%$ of 72 disease-free controls were positive for the antigen recognized by the $D 8 / 17$ antibody.

Unfortunately the supply of the single alloantiserum used by Patarroyo et al. in the original studies (10) has been exhausted making direct comparisons between the original antibody and the present monoclonal antibody impossible. However, preliminary studies with the original alloantiserum by Winchester and Kunkel (18) and Wang (19), indicated that the alloantiserum identified an antigen similar to the Ia class of MHC antigens. The antigen was further suggested to be the product of a gene within the MHC distinct from but possibly linked to those controlling the DR specificities. Our preliminary estimation of the MW of D8/17 (unpublished data) is $84,000 \mathrm{D}$ indicating that the corresponding antigens are probably unrelated.

Irrespective of these discrepancies, the cell-surface marker recognized by $\mathrm{D} 8 / 17$ thus far appears to be unique to B-lymphocytes since it was not present on $\mathrm{T}$ cells, macrophages, or non-B cell populations isolated from RF patients. Differential gradient studies and identification of the $B$ cell population with the appropriate markers indicates that of the $\mathrm{D} 8 / 17$ positive cells $>90 \%$ of these cells are B cells while most (though not all) of the $B$ cells of nonrheumatic individuals were negative. In addition, with the exception of the K-H lymphoblastoid line (DR specificity undefined), this marker was not present on a standard panel of eight lymphoblastoid lines prepared from local and workshop derived HLA-D homozygous disease-free donors. The fact that the marker was identified on $B$ cells from geographically disparate and ethnically diverse RF

Table V. A Comparison of HLA Types and Percent Positive B Cells in Rheumatic Fever Patients

\begin{tabular}{|c|c|c|c|c|c|c|c|}
\hline \multirow[b]{2}{*}{ Patient } & \multicolumn{6}{|c|}{ HLA phenotype } & \multirow{2}{*}{$\begin{array}{c}\% \text { B } \\
\text { cells } \\
\text { Positive }\end{array}$} \\
\hline & $\mathbf{A}$ & B & $\mathbf{C}$ & DR & DRW & DQW & \\
\hline Y.Z. & $24-29$ & $14-35$ & W4,w8 & 5,7 & $52-53$ & $3-2$ & 20 \\
\hline A.Z. & $24-29$ & $14-35$ & W4,w8 & 5,7 & $52-53$ & $3-2$ & 33 \\
\hline J.G. & $3-29$ & $7-$ & ND* & 4- & $53-$ & 3- & 20 \\
\hline R.S. & $23-11$ & $35-55$ & W3,w4 & $4-$ & $53-$ & $1-3$ & 43 \\
\hline E.G. & $2-$ & $44-8$ & w7 & $2-3$ & $52-$ & $1-2$ & 39 \\
\hline G.K. & $28-32$ & $14-44$ & ND & $1-$ & ND & $1-$ & 22 \\
\hline M.P. & $23-30$ & $13-50$ & W6 & $3-7$ & $52-53$ & $2-$ & 26 \\
\hline P.Y. & $2-11$ & $46-$ & W1,W3 & $8-5$ & $52-53$ & $3-$ & 20 \\
\hline
\end{tabular}

* Not done. 


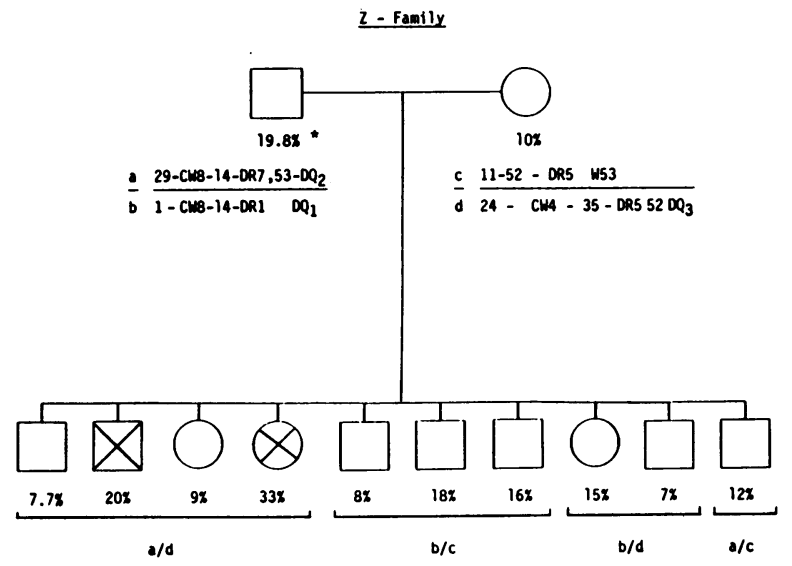

$\bigotimes \bigotimes \cdot$ rheumatic fever patients

- refers to $x$ positive cells

Figure 1. Pedigree of a family who had two RF patients, showing the percentage of positive B cells and HLA haplotypes. Note that four siblings had identical HLA haplotypes but only two had RF.

patients further supports the disease-specific nature of this marker compared to disease-free individuals.

The question of whether the elevated numbers of positive $B$ cells seen in RF individuals and their families was merely a reflection of a $B$ cell activation or differentiation antigen was carefully considered. Since nephritic patients were also exposed to a significant antigenic stimulus, (as measured both by a rise in antistreptococcal antibody titers and isolation of group A streptococci) the difference in results between rheumatic and nephritic patients and their families suggests that expression of the marker on a B cell is not solely the result of B cell activation by an undefined streptococcal antigen. Further support for this concept is the observation that this marker is present $25 \mathrm{yr}$ after the acute RF episode. For example, seven patients tested at least $20 \mathrm{yr}$ later after a single episode of RF with no clinical or laboratory evidence of an intervening streptococcal infection or RF recurrence had an average of $26 \%$ positive $B$ cells which is well above the normal range for

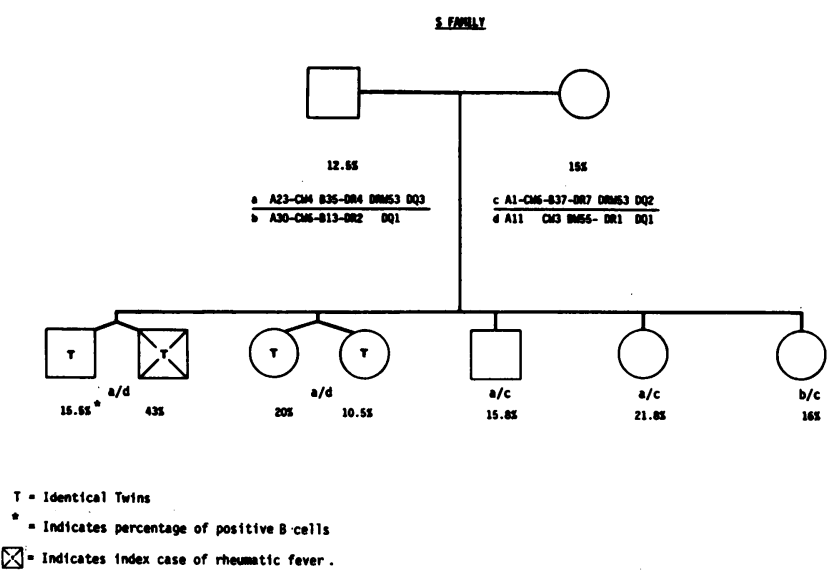

Figure 2. Pedigree of a RF family in which there were two sets of identical twins all with identical HLA haplotypes. Only one of the male identical twins had RF and exhibited the highest percentage of positive B cells.
D - Family - Nephritic

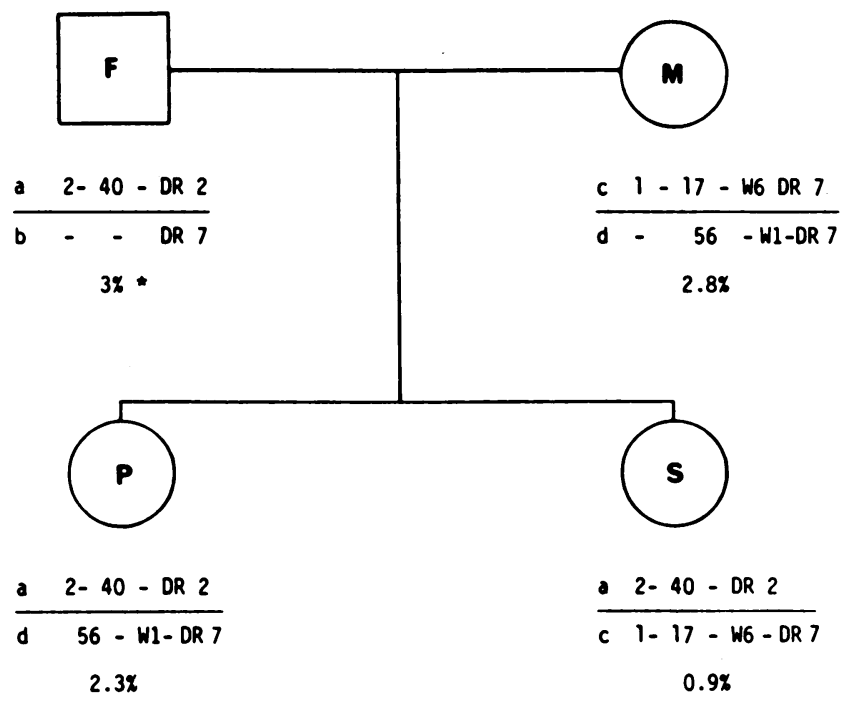

- indicates percentage of positive B-cells

$P \quad$ indicates index case of nephritis

Figure 3. Pedigree of a nephritic family in which one child had poststreptococcal glomerulonephritis. Note the very low percentage of positive $B$ cells in all members of the family irrespective of the haplotypes.

this age. In additional experiments (not shown), the marker was not expressed by B cells previously activated by mitogen Formalin Staphylococcus aureus or cytokine (interleukin 4), and there was no increased population of activated cells as assessed by cytofluorographic measurement of indirect immunofluorescence using $\mathrm{MAb}$ reactive with well-described $\mathrm{B}$ cell activation antigens such as Tac, $4 \mathrm{~F}_{2}, \mathrm{CD} 23$ (see Table IV). The alternative view is that the throat infection responsible for RF induces a B cell marker that is unique for this disease and is not seen in streptococcal infections causing nephritis.

The definition of positive and negative phenotypes allowed a preliminary investigation of the mode of inheritance of this putative genetic marker. An evaluation of the mechanism of inheritance, however, should include an understanding of the trait itself: is it an inherited characteristic as such, a true genetic marker, or does it result from the expansion of a normal subset of B lymphocytes under genetic control? The data indicate that there is considerable quantitative variation, even in identical twins, and more importantly, that the degree of expression of the trait is at least partly dependent on environmental influences. This is consistent with the observations that the affected individual regularly exhibits the highest expression of the phenotype in the respective sibship. Additionally, since all individuals have at least some cells expressing the marker, the D8/17 phenotype does not appear to be a conventional genetic marker. In spite of our lack of understanding of the nature of the trait and based on the essentially uniform positivity of healthy siblings in families with both parents positive 
for the trait, we hypothesize that this phenotype may reflect the expression of a recessive genotype although other forms of inheritance cannot be excluded.

Since most conventional cell antigens are dominantly inherited structural traits, this observation appears to support the view that the mechanisms of inheritance involve instead the genetic control of the number of B cells expressing D8/17. Given its obvious relationship to rheumatic disease, it will be necessary to establish whether the marker-defined subset of $B$ cells was already expanded prior to the time of the acute attack in the proband or whether the latter event caused its expansion. The presence of the trait in healthy siblings and even in a few persons unrelated to patients suggests that disease is neither necessary as a cause of the expansion nor is it a necessary consequence of it. These findings are consistent with our hypothesis that RF susceptible individuals have increased numbers of B cells expressing the D8/17 antigen before the disease onset, which are perhaps further increased during the actual disease secondary to the absence of normal regulatory mechanisms. This abnormal expression could then persist for years after the initial attack.

Similar mechanisms have been postulated for other diseases in which both genetic and environmental factors are required. Two examples are the autoimmune hepatitis that may follow infection with Schistosoma Japonicum (20) and insulin-dependent diabetes mellitis (IDDM) (21). Both examples, however, are associated with HLA antigens and one, IDDM, is inherited in linkage with the HLA genes. Neither association nor linkage to HLA are suggested by the limited data available thus far in this study.

However, it should be pointed out that Ayoub et al. (9) as well as Anastasiou-Nana et al. (22) recently reported a significant increase in the Class II alleles DR2 and DR4 in Black and Caucasian RF patients, respectively. Furthermore, Hafez et al. (23) showed a possible correlation with HLA B5 and RF susceptibility in detailed multiplex family studies of rheumatics and Jhinghan et al. (24) showed a close association with DR3 in affected individuals from North India. These observed differences could easily result from differences in the ethnic background or clinical selection of their study groups. It is also possible that certain of the allosera defining those particular HLA antigens might also contain an 883-like specificity reactive with the same B cell subset defined by the monoclonal antibody, D8/17, used in these studies.

If, as postulated, the monoclonal $\mathrm{D} 8 / 17$ is both highly specific and sensitive in its definition of a cell marker associated with susceptibility to RF, then the potential public health implications are significant. In this context our studies were concerned solely with patients who had had one established attack of RF and the predictive value of the marker was not tested. However in a recent study of RF patients and their families in New Zealand by Regelmann et al. (25) one unaffected individual in a rheumatic family who was markedly positive for the D8/17 marker went on to contract the disease. While this represents only a single case, and in an already affected sibship, it suggests that early identification of susceptible individuals in the general population could lead to more effective antibiotic strategies, thus enhancing the prevention of the disease. These studies are now in progress. Furthermore, in those individuals who develop subtle or nonspecific manifestations of the disease and who do not completely fulfill the Jones criteria, the presence of the marker might be useful in arriving at a diag- nosis and establishing preventative regimens. Finally, in view of the possibility of future streptococcal vaccines, individuals positive for the marker would be prime candidates for these vaccines if their excessive risk for RF could be demonstrated in prospective studies.

\section{Acknowledgments}

This research was supported in part by a General Clinical Research grant (MO1-RR00102) from the National Institutes of Health; American Heart Association, New York Affiliate; U. S. Public Health Service AI-18149, AM-13690; The Arthritis Foundation and its New York Chapter, and the J. M. Foundation.

\section{References}

1. Veasy, G., S. E. Wiedmeier, G. S. Orsmond, H. D. Ruttenberg, M. M. Boucek, S. J. Roth, V. F. Tait, J. A. Thompson, J. A. Daly, E. L. Kaplan, and H. R. Hill. 1987. Resurgence of acute rheumatic fever in the intermountain area of the United States. N. Engl. J. Med. 316:421-427.

2. Wald, E. R., B. Dashefsky, and D. Chiponis. 1986. Acute rheumatic fever in Western Pennsylvania and the Tri-state area. 26th Interscience Conference on Antimicrobial Agents and Chemotherapy, New Orleans, LA. 977:277. (Abstr.)

3. Zabriskie, J. B. 1986. Rheumatic fever: a model for the pathological consequences of microbial-host mimicry. Clin. Exp. Rheum. 4:65-73.

4. Cheadle, W. B. 1889. Harvean lectures on the various manifestations of the rheumatic state as exemplified in childhood and early life. Lancet. i:821-832.

5. Wilson, M. G., M. D. Schweitzer, and R. Lubschez. 1943. The familial epidemiology of rheumatic fever: genetic and epidemiological studies. J. Pediatr. 22:461.

6. Taranta, A., S. Toorsdag, J. D. Metrakos, W. Seigier, and I. Uchida. 1959. Rheumatic fever in monozygotic and dizygotic twins. Circulation. 20:778.

7. Falk, J. A., J. L. Fleischman, J. B. Zabriskie, and R. E. Falk. 1973. A study of HLA antigen phenotype in rheumatic fever and rheumatic heart disease patients. Tissue Ant. 3:173-178.

8. Murray, G. C., M. M. Monteil, and R. H. Persellin. 1978. A study of HLA antigens in adults with acute rheumatic fever. Arthritis Rheum. 21:652-656.

9. Ayoub, E. A., D. J. Barrett, N. K. Maclaren, and J. P. Krischer. 1986. Association of class II human histocompatibility leucocyte antigens with rheumatic fever. J. Clin. Invest. 77:2019-2026.

10. Patarroyo, M. E., R. J. Winchester, A. Vejerano, A. Gibofsky, F. Chalem, J. B. Zabriskie, and H. G. Kunkel. 1979. Association of a B-cell alloantigen with susceptibility to rheumatic fever. Nature (Lond.). 278:173-174.

11. Zabriskie, J. B., D. Lavency, R. C. Williams, S. M. Fu, C. A. Yeadon, M. Fotino, and D. G. Braun. 1985. Rheumatic fever associated B-cell alloantigen as identified by monoclonal antibodies. $A r$ thritis Rheum. 28:1047-1051.

12. 1984. Jones criteria (revised) for guidance in the diagnosis of rheumatic fever. Circulation. 69:204A-208A.

13. Boyum, A. 1966. Ficoll-hypaque method for separating mononuclear cells and granulocytes from human blood. Scand. J. Clin. Lab. Invest. (Suppl.) 77:57-62.

14. van de Putte, L. B. A., C. J. L. M. Moijer, G. J. Lafeber, R. Klienjan, and A. Cats. 1976. Lymphocytes in rheumatoid and non rheumatoid synovial fluid: non specificity of high $\mathrm{T}$ cell and low B-cell percentages. Ann. Rheum. Dis. 39:451-454.

15. Crow, M. K., and H. G. Kunkel. 1985. Activated B lymphocytes: stimulators of an augmented autologous mixed leucocyte reaction. Cell. Immunol. 90:555-568. 
16. Albert, E. D., M. P. Baur, and W. R. Mayr, editors. 1984. Histocompatibility testing. Springer Verlag, New York.

17. Freund, J. E. 1962. Mathematical Statistics. Prentice Hall, Englewood, NJ.

18. Winchester, R. J., and H. G. Kunkel. 1979. The human Ia system. Adv. Immunol. 27:258.

19. Wang, C. Y. 1979. Structural and functional characterization of surface antigens on human B lymphocytes. Ph.D. Thesis, The Rockefeller University. 55-63.

20. Hirayama, K., S. Matsushita, I. Kikuchi, M. Iuchi, N. Ohta, and T. Sasazuki. 1987. HLA-DQ is epistatic to HLA-DR in controlling the immune response to schistosomal antigen in humans. Nature (Lond.). 327:426-430.

21. Rubinstein, P., and S. Rodriguez de Cordoba. 1988. Insulindependent diabetes mellitis: genetic susceptibility, autoimmune components and environmental factors. In Clinical Aspects of Autoimmunity. In press.
22. Anastasiou-Nana, M. I., J. L. Anderson, J. F. Carlquist, and J. N. Nanas. 1986. HLA-DR typing and lymphocyte subset evaluation in rheumatic heart disease: A search for immune response factors. $\mathrm{Am}$. Heart J. 112:992-997.

23. Hafez, M., A. Chakravarti, El-Shennawy, Z. El-Morsi, S. H. El-Sallab, and Y. Al-Tonbary. 1985. HLA antigens and acute theumatic fever. Gen. Epidemiol. 2:273-282.

24. Jhinghan, B., K. S. Reddy, V. Taneja, M. C. Vaidya, and M. L. Bhatia. 1986. HLA, blood groups and secretor status in patients with established rhematic fever and rheumatic heart disease. Tissue Ant. 27:172-178.

25. Regelmann, W. E., E. D. Gray, R. Talbot, L. Cairns, D. Martin, L. C. Miller, J. B. Zabriskie, D. Braun, and S. S. Rich. 1987. Distribution and inheritance of "rheumatic" antigens in peripheral blood of RHD patients and their families. Xth Lancefield International Symposium on Streptococci and Streptococcal Diseases, 58:85. (Abstr.) 